\title{
DRIVING FAULT-TOLERANT CONTROL STRATEGY OF FOUR-WHEEL DRIVE ELECTRIC VEHICLE
}

\author{
Jing Wang ${ }^{1}$ \\ 1 Puyang Vocational and Technical College, Henan 457000, China. \\ Email: wangjinghenan2001@163.com
}

\begin{abstract}
Objective: To improve the safety and stability of the vehicle in the event of a fault by studying the drive fault-tolerant control strategy of a four-wheel drive electric vehicle. Methods: A hybrid non-singular terminal sliding mode control (HNTSM) controller is proposed by combining linear sliding mode control and non-singular terminal sliding mode, and its performance is compared with proportional-integral-derivative controller and linear sliding mode controller through simulation experiments. A torque distribution strategy is proposed based on the control distribution method, and simulation experiments are performed on the strategy. Finally, a hierarchical fault-tolerant controller is proposed, which divides the fault-tolerant system into upper and lower controllers to achieve different control goals. The controller is numerically simulated based on MATLAB/Simulink. Results: The instantaneous overshoot of HNTSM controller start-up is small, the current response is stable, and the optimization effect is the most significant. The torque distribution strategy can effectively follow the change of yaw angular velocity and ensure that the side deviation angle of the centre of mass does not exceed a certain range. The hierarchical fault-tolerant controller controls the vehicle, which can effectively track the speed and yaw rate; at the same time, it realizes the active steering of the front wheels to keep the vehicle turning at a constant speed. Conclusion: The three fault-tolerant control methods proposed in this study are feasible and excellent, which can effectively improve the safety and stability of automobile faults.
\end{abstract}

Keywords: Four-Wheel Drive Electric Vehicle; Fault-Tolerant Control Strategy; Hybrid NonSingular Terminal Sliding Mode Control; Torque Distribution Strategy; Hierarchical Fault-Tolerant Controller.

\section{Introduction}

Four-wheel drive vehicles increase the driving force by increasing the utilization of wheel adhesion. Researchers worldwide have focused on this technology, which has now become the research hot spot in the automotive field. Many well-known vehicle enterprises have applied four-wheel drive technology to the research, development, and practical applications of various sports vehicles and high-end vehicles [1].

A four-wheel drive electric vehicle, by installing four motors directly in the four-wheel hubs of the vehicle, makes it possible to omit a large number of automotive mechanical parts compared to a traditional vehicle, thereby simplifying the overall structure of the vehicle [2].

In addition, four-wheel-drive electric vehicles use new energy: the electrical energy; therefore, compared with traditional gasoline vehicles, fourwheel-drive electric vehicles have not only greater energy efficiency but also the advantage of clean energy and small pollution. Affected by the response performance of automobile motors, the control performance of four-wheel drive electric vehicles has also been improved to a certain extent [3].

The four wheels of a four-wheel drive electric vehicle are not connected with rigid mechanical devices. Thus, the running status of the wheels cannot interfere with each other, which greatly improves the transmission efficiency of the vehicle and reduces the overall quality of the vehicle, as well as simplifying the structure of the vehicle. Therefore, the distance travelled by electric vehicles can be extended, indicating the way forward in the field of later automotive research.

Since the four-wheel drive torque of a four-wheel drive electric vehicle is mutually independent, it is convenient to perform integrated linear control. However, when controlling the wheels, the failure of any mechanical component, such as the automobile sensor or inverter, will cause the corresponding wheel hub motor to malfunction, resulting in an error in the provided torque [4].

Also, the failure of any one or more in-wheel motors will have an impact on the performance of the four-wheel drive electric vehicle, reducing the safety and stability of the vehicle. Therefore, by 
studying the corresponding fault-tolerant control strategy of the vehicle, when the motor of the vehicle fails and the formal state of the vehicle is affected, the fault-tolerant control of the vehicle's motor failure is performed to ensure that the vehicle can complete operations such as continued steering, thereby improving the and the security stability of the vehicle [5].

In this study, a combination of linear sliding mode control and non-singular terminal sliding mode is proposed. A hybrid non-singular terminal sliding mode control (HNTSM) controller is proposed. At the same time, a torque distribution strategy and a hierarchical fault-tolerant controller are proposed. It is expected that through the above three controllers and control strategies, the vehicle can still run stably when a fault occurs.

The feasibility and superiority of the proposed method are verified by respective simulation experiments.

\section{Methods \\ 2.1 Hybrid Sliding Mode Control Strategy}

The general control system is often affected by various parameters, external interference, and noises, resulting in various changes in the structure. Therefore, sliding mode variable structure control was born based on this. This control was developed based on the discontinuity of the system, that is, the structure of the system will be adjusted at any time according to changes in various parameters and external conditions during operation [6].

HNTSM control is a complex sliding mode control. It is a new sliding mode controller that combines linear sliding mode and non-singular terminal sliding mode structures.

The non-singular terminal sliding mode controller avoids the singularity zone during the research and development of sliding mode and adds the nonlinear term to the sliding mode controller; therefore, the system state can converge to the expected value in the shortest time. However, since the convergence of the non-singular terminal sliding mode controller is not high enough, when the actual state of the system is far away from the equilibrium point, the response speed will be slower, the used convergence time gets longer, and the dynamic performance of the system is reduced. The linear sliding mode controller uses exponential deviation to achieve asymptotic convergence; therefore, the response performance is better. However, instead of being completely coincident with the given trajectory, it can only be infinitely close [7].

Therefore, to improve the control performance of sliding mode control system, this study combines linear sliding mode control with non-singular terminal sliding mode and proposes a HNTSM controller.
Through simulation experiments and the comparison with proportional-integral-derivative (PID) controller and the linear sliding mode (LSM) controller, its feasibility and superiority are analysed.

\subsection{Torque Distribution Strategy}

At present, most of the four-wheel independent drive electric vehicles adopt overdrive systems. A number of different control distribution methods have been developed globally to enable the wheel motor, thereby achieving the yaw torque $\triangle \mathrm{Mz}$ obtained by the control system. The simplest control distribution method is based on a hypothetical linear model. It is found that the result of this solution is not the optimal solution [8].

Therefore, researchers generally adopt the method of solving quadratic programming to obtain the optimal solution; also, the numerical methods are sometimes adopted. However, this method has significant disadvantages. The operation of this method requires a lot of computing resources, which is difficult to achieve real-time control.

For different optimization objectives, different objective functions need to be selected.

The evaluation of various control objectives worldwide has been studied. Most researchers have chosen the hybrid objectives of the driver itself and the error of the control effect [9].

Also, the control target selected in this study not only needs to realize that the four-wheel hub motors can track the total yaw torque but also has to reduce the torque output of the wheel motors of the automobile wheels, as well as making full use of the adhesion torque of each wheel.

To obtain the ideal yaw moment by controlling the distribution method, the wheel torque should not exceed the limit provided by the ground; otherwise, the wheel may slip. However, there is a strong coupling relationship between the longitudinal and lateral forces of the wheel, which can be observed through the force circle model of the wheel.

The mathematical model of the force circle of the wheel is:

$$
\left(\frac{F_{x}}{C_{x}}\right)^{2}+\left(\frac{F_{y}}{C_{y}}\right)^{2} \leq\left(\mu F_{z}\right)^{2}
$$

Where: $F_{x}$ and $F_{y}$ represent the longitudinal and lateral forces of the wheel; $C_{x}$ and $C_{y}$ represent the longitudinal and lateral stiffness of the wheel; $u$ represents the road surface adhesion coefficient.

This study improves the utilization of the road adhesion coefficient by controlling the slip ratio of the driving wheels, which improves the adhesion of the wheels to the road to a certain extent and also increases the lateral stability and safety of the vehicle when turning. 
The control objective equation proposed through research is:

$$
J=(1-\varepsilon)\left(F^{T} L-\Delta M_{Z}\right)^{T}\left(F^{T} L-\Delta M_{Z}\right)+\varepsilon F^{T} W F
$$

In the equation:

$$
F=\left[\begin{array}{llll}
\frac{T_{1}}{r_{\text {eff }}} & \frac{T_{2}}{r_{\text {eff }}} & \frac{T_{3}}{r_{\text {eff }}} & \frac{T_{4}}{r_{\text {eff }}}
\end{array}\right]^{T}, L=\left[\begin{array}{llll}
-l_{1} & l_{2} & -l_{3} & l_{4}
\end{array}\right]^{T}
$$

$$
W=\operatorname{diag}\left(\frac{\sum_{i=1}^{4}\left(\mu^{2} F_{z i}^{2}-F_{y i}^{2}\right)}{\mu^{2} F_{z 1}^{2}-F_{y 1}^{2}}, \frac{\sum_{i=1}^{4}\left(\mu^{2} F_{z i}^{2}-F_{y i}^{2}\right)}{\mu^{2} F_{z 2}^{2}-F_{y 2}^{2}}, \frac{\sum_{i=1}^{4}\left(\mu^{2} F_{z i}^{2}-F_{y i}^{2}\right)}{\mu^{2} F_{z 3}^{2}-F_{y 3}^{2}}, \frac{\sum_{i=1}^{4}\left(\mu^{2} F_{z i}^{2}-F_{y i}^{2}\right)}{\mu^{2} F_{z 4}^{2}-F_{y 4}^{2}}\right)
$$

Where: $\mathrm{Ti}(\mathrm{i}=1,2,3,4)$ represents the torque provided by the four-wheel hub motor. $r_{\text {eff }}$ represents the effective radius of the vehicle wheel. $F$ represents the error between the yaw moment calculated by the controller and the actual yaw moment. W stands for reducing the longitudinal force of the vehicle wheel, and $\varepsilon$ stands for a constant used to balance the distribution error and control cost.

The weight matrix $\mathrm{W}$ in the penalty function is used to scale the adhesion of the four wheels. For example, $\mu^{2} F_{z i}^{2}-F_{y i}^{2} \leq 0$ means that there is no adhesion remaining on the $\mathrm{i}$-th wheel. At this time, the corresponding $\mathrm{W}$ will set a huge number to "punish" the i-th wheel to reduce the adhesion of the wheel.

The linear equation can find the optimal wheel force distribution. Therefore, the problem of control distribution is transformed into a constrained quadratic programming problem, and the obtained motor torque is input into the vehicle model for experimental simulation.

\subsection{Hierarchical Fault-tolerant Controller}

This section uses a hierarchical control system to design fault-tolerant controllers. The fault-tolerant controllers are divided into upper and lower controllers. The upper controller is used to control the dynamics model of the entire vehicle and the driving speed of the vehicle as close to the expected value as possible. The lower controller is used to control the torque of the three normal wheels except for the fault and the yaw rate of the vehicle as close to the expected value as possible, thereby achieving the stable operation of the vehicle.

Upper controller: To control the vehicle dynamics of the vehicle and weigh the limits of the actual torque, the upper controller uses a nonlinear model predictive control (NMPC) algorithm. On the one hand, NMPC meets the requirements of model predictive control theory for the accuracy of the model of the control object, facilitates the process description, and has a large amount of information redundancy, which increases the robustness and stability of the system. On the other hand, NMPC uses a rolling optimization strategy.
Compared with the global one-time optimization strategy, the rolling optimization strategy can adjust the instability caused by model distortion, interference, mismatch, and other factors in time, which has excellent dynamic performance. The NMPC algorithm is easier to be applied to non-linear, large delay, constrained, non-minimum phase and other practical processes, and can more effectively solve the multi-variable and constrained problems [10]. Therefore, it is reasonable to use this method to design the upper controller.

When the wheel hub motor of any of the wheels fails during the running of the vehicle, the wheel of the vehicle will no longer provide torque, and the slip rate of the wheel will also be zero. Therefore, if the wheel hub motor of any wheels fails, it is only necessary to set the maximum and minimum values of the constraint of the slip ratio of the wheel to 0 .

Lower controller: This study uses the lower controller to output the torque of three wheels to control the yaw rate of the wheels. It is necessary to track the virtual control input of the upper controller, that is, the wheel slip rate output by the upper controller. The dynamic model of the wheel is simpler than the dynamic model of the vehicle; thus, only a PID controller needs to be designed.

In this study, MATLAB/Simulink is used to simulate the fault-tolerant control algorithm proposed above. The simulation experiments are performed with the vehicle during constant speed steering. The vehicle is in a constant speed steering operating state, which means that the vehicle driver only operates the vehicle's steering wheel and does not control the drive of the vehicle. At the same time, the vehicle always maintains the initial degree of constant speed. In this driving state, taking the left front wheel of the vehicle as an example, the following three scenarios are compared with simulation experiments to verify the effectiveness of the proposed fault-tolerance control strategy:

Scenario 1: No-drive scenario.

This scenario simulates a vehicle without any vehicle dynamics control strategy applied. In the simulation experiment, the torque on all wheels is set to zero when the vehicle is turning.

Scenario 2: Two-wheel drive scenario.

This scenario simulates a situation where the motor of a wheel fails and the motor torque of the opposing wheel is set to 0 . 
At this time, only the motors of two front wheels or two rear wheels provide torque, and the fourwheel drive becomes two-wheel drive.

Scenario 3: Three-wheel drive scenario.

This scenario simulates the situation in where the three wheels normally provide torque, which is the focus of this study.

The torque of the motor of the failed wheel is set to 0 , and the torque of the other three wheels is distributed by using a hierarchical fault-tolerance controller.

\section{Results and Discussions}

\subsection{Simulation Results of HNTSM Controller}

The simulation experiment uses the detection of the motor current signal. Whether the driving motor of the vehicle is in a fault state is determined, and different current controllers are used according to the obtained monitoring conditions to output a current response signal after the current is controlled.

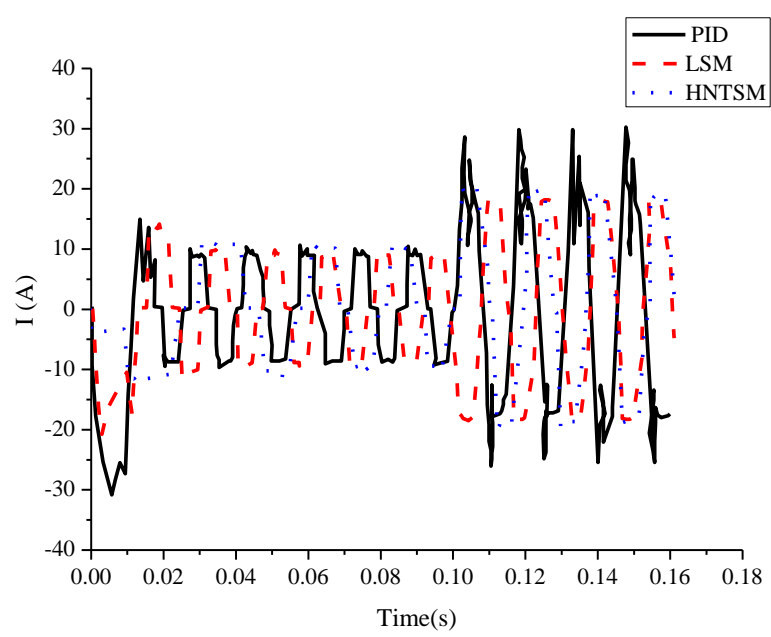

Figure 1: Comparison of current response of three controllers

Figure 1 is a comparison of the current response of a PID controller, a linear sliding mode controller, and a controller. As shown in Figure 1, although the current response of the PID controller is small, the overshoot in the initial stage of the response is large, and a large spike is generated. Although the LSM controller generates smaller spikes and the current response is more stable, it still cannot avoid a large current overshoot during startup. Compared with the first two controllers, the instantaneous overshoot of the non-singular terminal sliding mode controller is small, the current response is stable, and the optimization effect is the most significant, which proves that the controller is feasible and excellent.

\subsection{Simulation Results of Control Allocation}

To prove the feasibility and superiority of the proposed control allocation strategy proposed above, in this study, SIMULINK and CARSIM are used for joint simulation, and line-shift simulation and J-turn simulation are performed simultaneously.

(1) Line shift simulation

The vehicle runs at a speed of $40 \mathrm{~m} / \mathrm{s}$. The front wheel of the vehicle has a sinusoidal input of $0.3 \mathrm{rad}$ and the period is $2 \mathrm{~s}$. Vehicle simulation of lane change is performed. The centroid yaw angle and yaw angular velocity of the vehicle are shown in Figures 2 and 3.

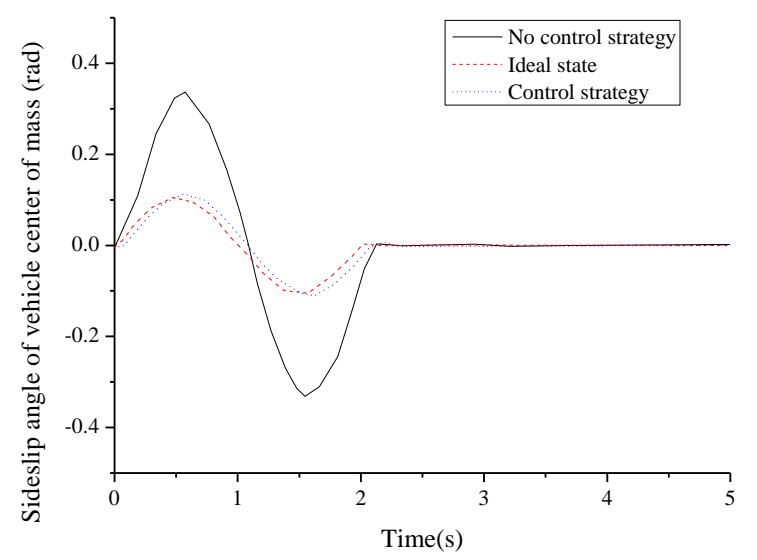

Figure 2: Centroid sideslip angle

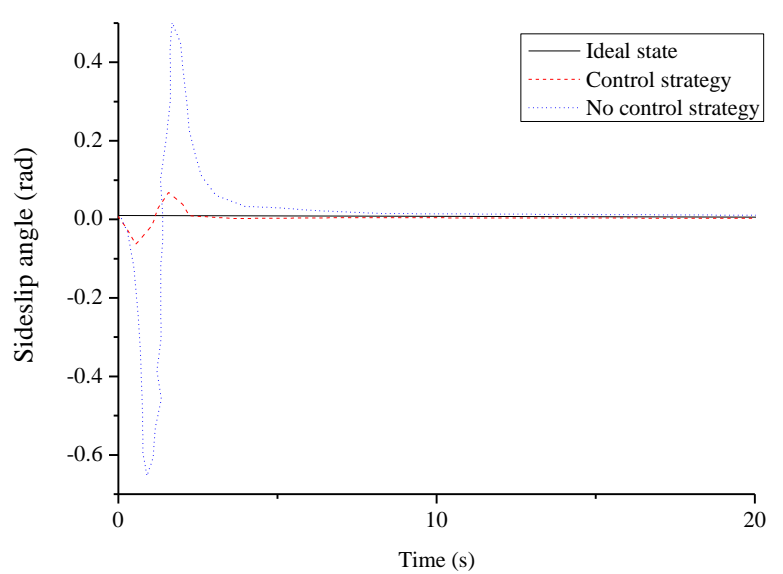

Figure 3: Yaw angular velocity

As shown in Figures 2 and 3, in the absence of a control strategy for the vehicle, the yaw angular velocity and the centroid sideslip angle of the vehicle are significantly different from the ideal state, resulting in the running direction of the vehicle deviating from the ideal direction of the vehicle. Under the same conditions, an electric vehicle with a control strategy is added. When the vehicle is changing lanes, due to the operation of the added control strategy, the yaw angular velocity of the vehicle is greatly increased, and the increase of the centroid sideslip angle is suppressed. 
The feasibility and goodness of the control allocation strategy can be seen from the results of the simulation experiment.

The control strategy can effectively suppress the change of yaw angular velocity and control the centroid sideslip angle within a certain range, which greatly improves the stability and safety of the vehicle.

(2) J-turn simulation of vehicles

After the vehicle is driven at an initial speed of 40 $\mathrm{m} / \mathrm{s}$ for $1 \mathrm{~s}$, the step of the universal disk is $0.1 \mathrm{rad}$.

The simulation results are shown in Figures 4 and 5 .

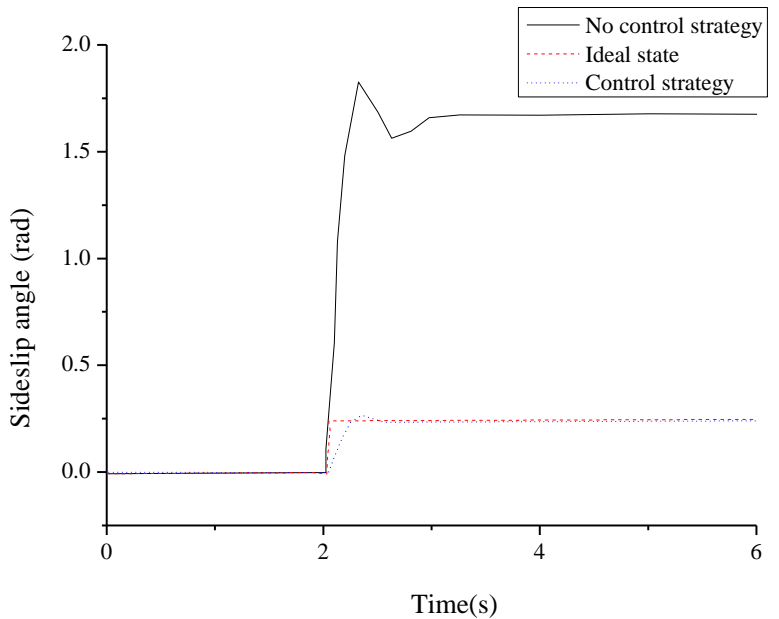

Figure 4: Yaw angular velocity of the vehicle

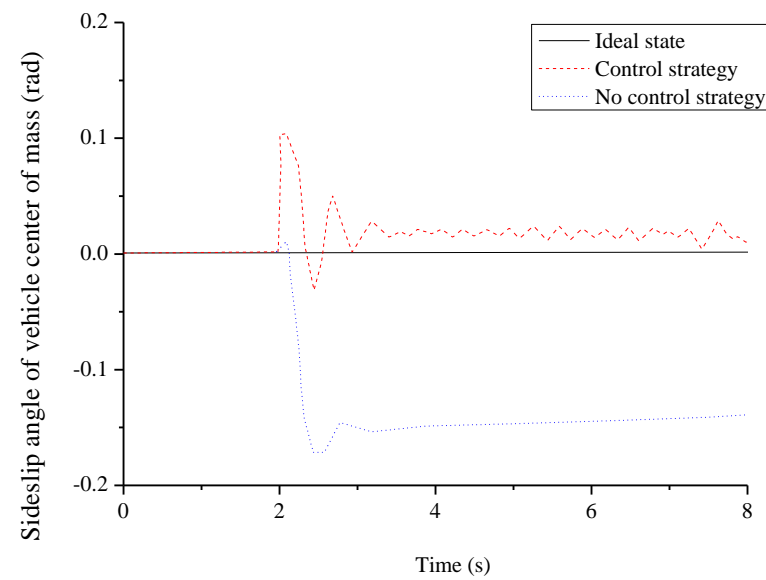

Figure 5: Centroid sideslip angle of the vehicle

As shown in Figures 4 and 5, under the condition that no control strategy is applied, the degree of yaw angular velocity of the vehicle during operation deviating from the ideal yaw angular velocity is large, and the deviation rate of the centroid sideslip angle of the vehicle is also significant. After the control strategy is applied, the deviation between the driving direction of the vehicle and the ideal driving direction is significantly reduced.

The feasibility and goodness of the control allocation strategy can be seen from the results of the simulation experiment.
The control strategy can effectively suppress the change of yaw angular velocity and control the centroid sideslip angle within a certain range, which greatly improves the stability and safety of the vehicle.

\subsection{Simulation Results of Hierarchical Fault- tolerant Controller}

Figure 6 shows the comparison of the running speed and the expected speed of the vehicle in three different scenarios. As shown in Figure 6, when the vehicle is not driven, its running speed is stable in the first 3 seconds. Then, it drops sharply from the third second, with a poor steady-state. When the vehicle is driving with two wheels, its driving speed remains unchanged for the first 2 seconds; then, it drops sharply from the second second, with a poor steady-state.

However, when the vehicle is driving under three-wheel drive, its driving speed remains unchanged in the first 2 seconds, but it changes slightly from the second second, and finally coincides with the expected speed at about $4.5 \mathrm{~s}$; also, it can maintain this running speed for stable driving.

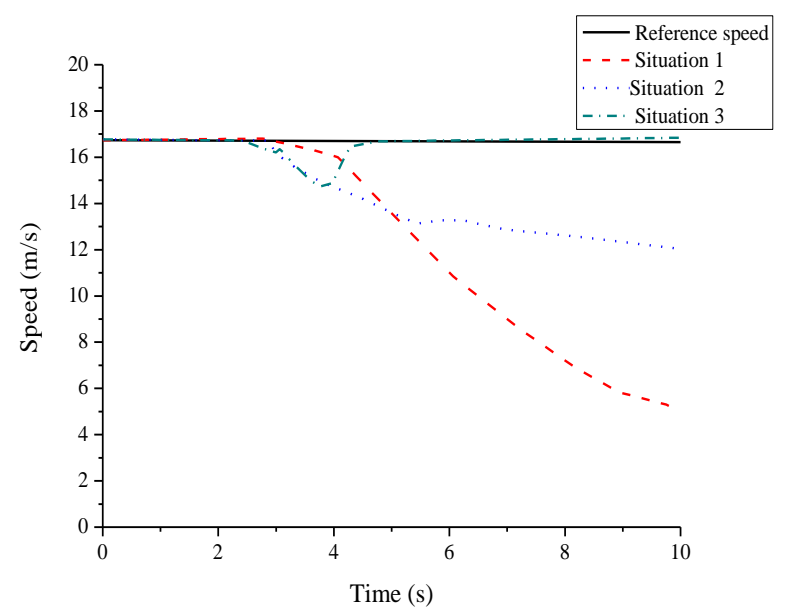

Figure 6: Comparison of running speed and expected speed

Figure 7 shows the comparison of the sideslip angle of the vehicle in three different scenarios.

As shown in Figure 7, when the vehicle is driven with no drive, its sideslip angle can be maintained at 0 in the first 2 seconds. However, the sideslip angle of the vehicle has a significant deviation from the second second; when the simulation experiment ends, it is still in a changing state; thus, the vehicle has always been deviated. When the vehicle is under two-wheel drive, its sideslip angle can also be maintained at 0 in the first 2 seconds; then, a large shock is generated, which eventually approaches 0 , making the vehicle gradually stable.

When the vehicle is driving under three-wheel drive, its sideslip angle can also be maintained at 0 in the first 2 seconds; then, a small amplitude shock is 
generated, which finally approaches 0 in about 6 seconds.

It can be seen from the comparison that although the sideslip angle of the vehicle oscillates during three-wheel drive, compared to the other two scenarios, the time required for the sideslip angle to stabilize to 0 is shorter than the time required for two-wheel drive scenario, indicating that the threewheel drive makes it easier to keep the vehicle stable.

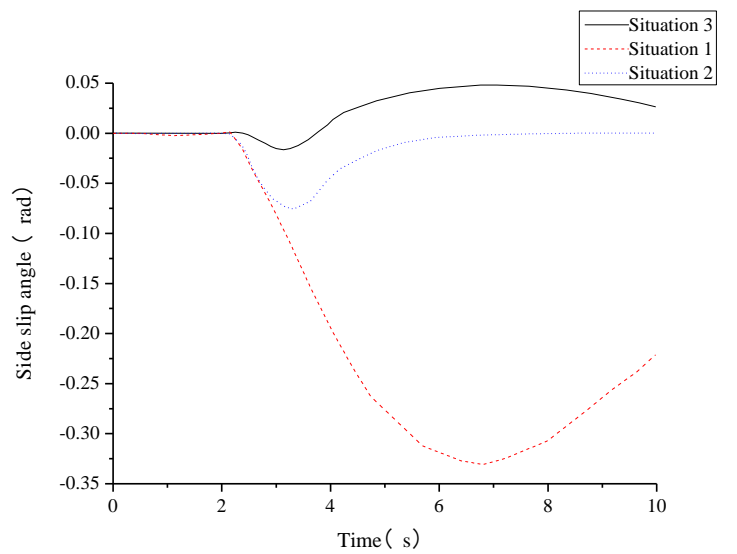

Figure 7: Comparison of the sideslip angles of vehicle

Figure 8 shows the comparison of the tracking effect of the running yaw rate under three different scenarios of the vehicle. As shown in Figure 8, the expected yaw rate of the vehicle remains 0 in the first 2 seconds. Then, two large changes occurred from $2 \mathrm{~s}$ to $4 \mathrm{~s}$, but it eventually stabilizes at about 4 seconds. When the vehicle is driven with no drive, the yaw rate of the vehicle can be maintained at zero in the first 2 seconds. However, a small oscillation occurs from 2 to 9 seconds, and the vehicle cannot accurately maintain the desired yaw rate. When the vehicle is driven under two-wheel drive, the yaw rate of the vehicle can also be maintained at 0 in the first 2 seconds, but a small amplitude oscillation occurs between $2 \mathrm{~s}$ and $10 \mathrm{~s}$. Finally, it approaches 0 in about 10 seconds.

The vehicle cannot accurately maintain the desired yaw rate. When the vehicle is driven under three-wheel drive, the yaw rate of the vehicle can be maintained at 0 in the first 2 seconds, and the changes in yaw rate can be accurately tracked between 2 seconds and 4.5 seconds.

Finally, it gradually approaches 0 and remains stable after 4.5 seconds.

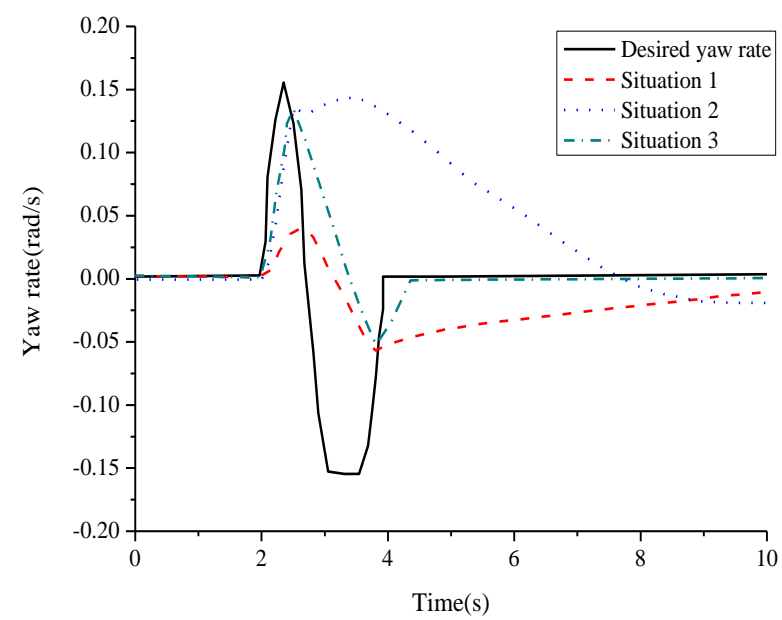

Figure 8: Comparison of vehicle yaw rat

In summary, by using the fault-tolerant controller designed in this study to control the vehicle, that is, simulation scenario 3 , the vehicle can effectively track the speed and yaw rate, as well as maintaining the stability of itself.

Therefore, the vehicle is kept turning at a constant speed, and the control effect is better than that of directly setting the opposite wheel torque of the faulty wheel to 0 .

\section{Conclusions}

Based on the previous experiences, a combination of linear sliding mode control and non-singular terminal sliding mode is proposed.

A HNTSM controller is proposed. At the same time, a torque distribution strategy and a hierarchical fault-tolerant controller are proposed. Then, simulation experiments are performed on three fault-tolerant strategies.

The experimental results show that the three fault-tolerant strategies proposed in this study have high feasibility and optimality, which can be applied to drive fault-tolerant control of four-wheel drive electric vehicles and can greatly improve the stability and safety of the vehicles.

However, there are some shortcomings in this study. Although the above three fault-tolerant strategies are effective, they cannot achieve completely accurate vehicle control, and there are still some errors. Therefore, in the subsequent study, it is necessary to improve the control strategy and consider combining various control strategies to improve the stability and safety of the vehicles. 


\section{References}

[1] Y. Luo, J. Luo, and Z. Qin,"Model-independent self-tuning fault-tolerant control method for 4WID EV,"International Journal of Automotive Technology,vol. 17, no. 6, pp. 1091-1100,2016

[2] Y. Fan, X. Zhang, M. Wei, et al.,"Fault-tolerant control for open-winding permanent magnet compact in-wheel motor drive system,"vol. 30, no. 2, pp. 98-105, 2015.

[3] C. A. Hu, H. Jing, R. R. Wang, et al.,"FaultTolerant Control of FWIA Electric Ground Vehicles with Differential Drive Assisted Steering,"IfacPapersonline, vol. 48, no. 21, pp. 1180-1185, 2015.

[4] B. A. Hechmi, M. Mongi, J. Mohamed, et al.,"Implementation of Improved Sliding Mode Observer and Fault Tolerant Control for a PMSM Drive,"Journal of Circuits Systems \& Computers, vol. 26, no. 2, pp. 1750032, 2016.

[5] W. Ding, Y. F. Hu, and L. M. Wu,"Investigation and Experimental Test of Fault-Tolerant Operation of a Mutually Coupled Dual Three-
Phase SRM Drive Under Faulty Conditions,"vol. 30, no. 12, pp. 6857-6872, 2015.

[6] Y. X. Xu, H. Yan, and J. B. Zou, "A Fault-Tolerant Control Strategy for Six-Phase Transverse Flux Tubular PMLM Based on Synthetic Vector Method,"IEEE Transactions on Plasma Science, vol. 43, no. 5,pp. 1332-1338, 2015.

[7] M. S. Keating, M. Pachter, and C. H. Houpis, "FAULT TOLERANT CONTROL SYSTEM: QFT DESIGN,”vol. 7, no. 6,pp. 551-559, 2015.

[8] Y. Shen, X. Bing, X. S. Ding, et al.,"A Review on Recent Development of Spacecraft Attitude Fault Tolerant Control System,"IEEE Transactions on Industrial Electronics,vol. 63, no. 5,pp. 1-1, 2016.

[9] T. Wang, H. Gao, and J. Qiu, "A Combined Fault Tolerant and Predictive Control for NetworkBased Industrial Processes," vol. 63, no. 4,pp. 2529-2536, 2016.

[10] L. Liu, Z. S. Wang, and H. G. Zhang,“Adaptive NN fault-tolerant control for discrete-time systems in triangular forms with actuator fault,"vol. 152, no. C,pp. 209-221, 2015. 